\title{
reviscafuences
}

ISSN: 1575.7072 | 8.15SN: 2172.7775

Páginas: $230-243$

Recibido: 2020-07-29

Revisado: $2020-11-13$

Aceptado: 2021-02-04

Preprint: 2021-05-01

Publicación Final: 2021-05-15

www.revistascientificas.us.es/index.php/fuentes/index

DOI: https://doi.org/10.12795/revistafuentes.2021.12307

\section{Mapeo sistemático sobre la aplicación de la obra de Pierre Bourdieu en la Educación Superior}

\author{
Systematic mapping on the application of the work of Pierre Bourdieu in Higher \\ Education
}

\begin{abstract}
La obra de Bourdieu, en especial los conceptos campo, capital y habitus han trascendido en las ciencias sociales para comprender lo que sucede en el proceso educativo. En este artículo se presentan los resultados de un mapeo sistemático desde el criterio metodológico de Petersen et al. (2008) para identificar la aplicación bibliográfica de estos conceptos en la Educación Superior. Se analizaron 104 documentos alojados en las bases bibliográficas Scopus y Web of Science entre 2009-2019. Las variables de estudio fueron los idiomas, países, accesibilidad, tipos de trabajo, trabajos de mayor impacto y líneas de investigación. Se encontró que existen algunas investigaciones sobre el tema, con un número reducido de documentos en acceso abierto, la mayor parte se encuentra en inglés y proviene de Reino Unido. Las líneas de investigación resultaron ser variadas, con una tendencia a estudiar los principios de elección y las trayectorias de los estudiantes, las prácticas docentes, la influencia del habitus y el capital en la transición del campo universitario al laboral, así como los estudios de género. Se concluye que el mapeo elaborado ofrece a los investigadores una selección detallada de la producción científica de alto impacto internacional que puede servir para determinar sus rutas investigativas.
\end{abstract}

Resumen

\section{Abstract}

Bourdieu's work, especially the concepts of field, capital and habitus, have transcended in the social sciences to understand what happens in the educational process. This article presents the results of a systematic mapping from the methodological criteria of Petersen et al. (2008) to identify the bibliographic application of these concepts in Higher Education. 104 documents hosted in the Scopus and Web of Science bibliographic databases between 2009-2019 were analyzed. The study variables were languages, countries, accessibility, types of work, jobs with the greatest impact and lines of research. It was found that there is some research on the subject, with a small number of open access documents, most of it in English and from the United Kingdom. The lines of research turned out to be varied, with a tendency to study the principles of choice and the trajectories of students, teaching practices, the influence of habitus and capital in the transition from university to work, as well as gender studies. . It is concluded that the elaborated mapping offers researchers a detailed selection of the scientific production of high international impact that can be used to determine their research routes.

\section{Palabras clave}

Bibliografía, Bourdieu, campo, capital, habitus, investigación, educación superior, mapa.

\section{Keywords}

Bibliography, Bourdieu, field, capital, habitus, research, higher education, map. 


\section{Introducción}

El arreglo conceptual para comprender las prácticas sociales (Campo + [Capital + Habitus]) formulado por el sociólogo bearnés Pierre Bourdieu ha sido ampliamente utilizado como un planteamiento teórico-metodológico en las ciencias sociales para abordar las complejidades de la práctica escolar, autores como Casillas et al. (2014), Salado et al. (2016), Lee y Chen (2017), y Singh et al., (2018), han realizado estudios desde el enfoque bourdiano en donde retoman "la relación de un cuerpo socializado (habitus) con el entorno objetivo de relaciones sociales (campo), y está mediada por las entidades allí circulantes (dispositivos) que pueden o no ser apreciados como recursos, es decir, como capital de cualquier especie" (Cerón, 2012:6).

Otros autores, se han dado a la tarea de formular interpretaciones acerca de las lecciones teóricas de Bourdieu (Bustamante, 2016; Cerón \& Galán, 2017; Ignatow \& Robinson, 2017; Bonello et al., 2018; Cruz, 2018; Salado \& Ramírez, 2018; Wacquant, 2018; Cerón, 2019; Hart, 2019) en las que construyen revisiones de la obra del autor para posicionar desde diversas aristas los nodos conceptuales campo, habitus y capital. De esta forma, las practicas sociales se presentan como un intento de comprensión sobre lo que las personas realizan de forma cotidiana, permanente y duradera. Si bien el objetivo de este articulo no es contribuir con una reconceptualización de la propuesta teórica de Bourdieu, si es necesario abordar brevemente los conceptos que la sustentan.

\subsection{Noción acerca del campo}

Bourdieu (1998:113) mencionó que "es posible construir un espacio cuyas tres dimensiones fundamentales estarían definidas por el volumen del capital, la estructura del capital y la evolución en el tiempo de estas dos propiedades", a este espacio lo llamo campo. Para Bourdieu la noción de campo delimita un espacio social donde ocurren una gama de interacciones entre los individuos en torno a bienes materiales o simbólicos. Los individuos que concurren en el campo tienen posiciones desiguales que se apoyan a su vez en las dotaciones distintas de capitales con que cuentan, lo que genera relaciones de dominación o conflicto (Alonso, 2002). Con base en esta noción, es posible suponer que la Educación Superior, constituye un campo, en el que participan individuos con relaciones de autoridad y compromiso producidas por las diferentes acumulaciones de capitales adquiridos durante la trayectoria académica.

\subsection{Noción acerca del capital}

Para Marx, el capital es una relación social de producción, donde existe una correspondencia asimétrica de las diferencias económicas entre quienes son propietarios de los medios de producción y quienes emplean la fuerza de trabajo para producir los bienes, de esta forma el capital se constituye como el dinero que se invierte en un proceso de producción para obtener al final de éste la cantidad invertida más una cantidad adicional.

Pierre Bourdieu recupera la noción marxista de capital desde el enfoque de los bienes desigualmente distribuidos, pero incluye esferas sociales no consideradas por Marx (Giménez, 1999). Para Bourdieu el capital se constituye por bienes materiales o simbólicos escasos, valorados en campos sociales determinados. El autor observó al arte, la religión o la escuela como campos en los que existen distintos bienes en disputa otorgó importancia a las formas de capital inmaterial como la cultura o el prestigio, lo que ha posibilitado entender que existen intercambios que rebasan la noción económica, y que estos se manifiestan en contextos complejos de socialización y valoración cultural, en el que, desde luego, la acumulación de dinero es importante (Grenfell, 2012).

Asimismo, enfatiza que el capital, al que llama cultural (que se presenta en tres formas: incorporado, objetivado e institucionalizado) es la especie de capital más eficiente en el mundo de la cultura y la educación (Bourdieu, 2001), ya que en el campo educativo no basta tener dinero o detentar poderes políticos para lograr el aprendizaje, sino que se requieren conocimientos y formas de comportamiento que se aprenden mediante largos procesos de socialización en la propia escuela y generan hábitos de vida y competencias específicas.

\subsection{La noción de habitus}

Al igual que existe una distribución desigual de los capitales, también hay una distribución desigual en la forma en la que se apropian y ponen en juego esos capitales en un campo. A este desequilibrio social definido por las prácticas cotidianas y duraderas de los sujetos Bourdieu le llamó habitus, es decir, es un "producto de la interiorización de los principios de una arbitrariedad cultural capaz de perpetuarse una vez terminada la acción pedagógica" (Bourdieu \& Passeron 1996:72). 
En términos sencillos el habitus es la intuición, el conocimiento experto de las personas que les permite conducirse en la sociedad de formas muy distintas a sus iguales, es un "conocimiento práctico que no contiene el conocimiento de sus propios principios" (Bourdieu 2012:243), sin embargo, el habitus no opera como una variable definitoria de la acción del ser, este se modifica por las experiencias, la acumulación de capitales en la trayectoria de vida y las posiciones del sujeto en un campo determinado (Bourdieu \& Wacquant 2005).

La producción científica que retoma estos tres conceptos es numerosa, una búsqueda simple de las palabras field, capital y habitus, en bases de datos como Scopus o Web of Science (WOS) que son consideradas de alto impacto internacional (Delgado \& Repiso, 2013), arroja más de 700 documentos, por otra parte, si se busca solamente la palabra habitus el volumen de información se eleva a más de 7,800 artículos. Lo anterior, origina el objetivo general de esta investigación, que es caracterizar la producción científica sobre los conceptos nodales de la obra de Pierre Bourdieu en el nivel educativo de la Educación Superior por medio de un mapeo sistemático de la literatura, en el que se trazan cuatro objetivos específicos: 1) identificar la producción científica entre 2009 y 2019,2 ) identificar los idiomas y los países en los que la investigación científica ha analizado el campo, el capital y el habitus, 3) caracterizar los documentos en cuanto a su accesibilidad y tipo de producción, 4) identificar los trabajos de mayor impacto internacional, y 5) determinar que líneas de investigación se han desarrollado en torno a los conceptos mencionados.

\section{Metodología}

Se realizó un mapeo sistemático de la literatura basado en la propuesta metodológica de Petersen et al. (2008). Esta metodología ha sido utilizada por diversos autores (López et al., 2015; Pérez \& Vladimirovna 2017; De las Heras et al., 2018; Ramírez \& García, 2018; Cantú et al., 2019) que coinciden en señalar que las etapas más importantes para realizar un estudio de esta naturaleza son: 1) definición de preguntas de investigación, 2) localización de la producción científica, 3) depuración de la producción científica, 4) creación y ajuste de una base de datos bibliográfica, y 5) análisis de la información. A continuación, se describe el proceso que se llevó a cabo:

\subsection{Definición de preguntas de investigación}

La primera etapa consiste en definir las preguntas que orientarán la investigación, debido a que el propósito de este trabajo es conocer la producción científica relacionada con los conceptos campo, capital y habitus, y su relación con la Educación Superior se plantearon los siguientes cuestionamientos:

- Q1 ¿Cuántos trabajos científicos se han producido respecto al tema de campo, capital y habitus en el contexto de la Educación Superior entre 2009 y 2019 ?

- Q2 ¿En qué países y en qué idiomas se produce la investigación sobre el tema?

- Q3 ¿Qué tipo de productos científicos se han publicado y cómo se caracteriza su accesibilidad para los lectores?

- Q4 ¿Qué trabajos han tenido mayor impacto en la producción científica internacional?

- Q5 ¿Qué líneas de investigación se han desarrollado respecto al tema?

\subsection{Localización de la producción científica}

La segunda etapa consistió en localizar los documentos científicos en Scopus y Web of Science (WoS). Se tomó la decisión de utilizarlas debido a que son dos de las principales bases de datos bibliográficas multidisciplinarias y de mayor impacto internacional (Cantú et al. 2019). Se realizó una búsqueda con la restricción temporal que abarca los últimos 10 años (2009-2019). Los términos utilizados fueron field, capital y habitus como elementos principales y higher education como elemento contextual. Se filtraron los resultados con base en los siguientes indicadores: 1) que se ubicaran en el campo de las ciencias sociales (social sciences en Scopus; education scientific disciplines y social sciences interdisciplinary en WOS), y 2) que fueran documentos en versión final. En la Tabla 1 se puede observar la cadena de búsqueda general que se confeccionó.

\subsection{Depuración de la producción científica}

En esta etapa se llevó a cabo una refinación de la búsqueda realizada, lo que significó prescindir de trabajos que aparecieron en ambas bases de datos, los trabajos sin resumen disponible en Scopus y WOS, así como 
Tabla 1

Cadena de búsqueda original

( TITLE-ABS-KEY ( field AND habitus AND capital ) AND TITLE-ABS-KEY ( higher AND education ) OR

TITLE-ABS-KEY ( university ) ) AND ( LIMIT-TO ( PUBYEAR , 2019 ) OR LIMIT-TO ( PUBYEAR , 2018 ) OR LIMIT-TO ( PUBYEAR , 2017 ) OR LIMIT-TO ( PUBYEAR , 2016 ) OR LIMIT-TO ( PUBYEAR, 2015 ) OR LIMIT-TO ( PUBYEAR , 2014 ) OR LIMIT-TO ( PUBYEAR , 2013 ) OR LIMIT-TO ( PUBYEAR , 2012 ) OR LIMIT-TO ( PUBYEAR , 2011 ) OR LIMIT-TO ( PUBYEAR , 2010 ) OR LIMIT-TO ( PUBYEAR , 2009 ) ) AND ( LIMIT-TO ( PUBSTAGE , "final") ) AND ( LIMIT-TO ( SUBJAREA , "SOCI" ) )

documentos como editoriales o erratas. En la Tabla 2 puede observarse la progresión para reducir la cantidad de documentos.

Tabla 2

Depuración de la producción científica

\begin{tabular}{l|l|l|l|} 
Criterios de exclusión & Scopus & WOS & Total \\
Cadena original & 140 & 171 & 311 \\
Documentos duplicados & 62 & & 187 \\
Documentos sin resumen & 11 & 25 & 151 \\
Documentos no académicos & 9 & 38 & 104 \\
Cantidad final & & & 104 \\
\hline
\end{tabular}

Adicionalmente se emplearon los siguientes criterios de inclusión $(\mathrm{Cl})$ y exclusión (CE) para poder seleccionar los documentos que mejor se ajustaran para responder las preguntas de investigación:

Criterios de inclusión $(\mathrm{Cl})$ :

- Cl1. Los documentos contienen una propuesta relacionada con el campo, capital y habitus desde el enfoque de Pierre Bourdieu.

- $\quad \mathrm{Cl}$ 2. Los trabajos se abordan desde la disciplina de las ciencias sociales.

- Cl3. Los documentos se publicaron en revistas, libros o conferencias que tienen como criterio de calidad la revisión por pares.

Criterios de exclusión (CE):

- CE1. Los documentos reflejan ideas que provienen de grupos políticos o gubernamentales.

- CE2. Los trabajos apoyan conceptualizaciones alejadas de la propuesta teórico-metodológica del campo, capital y habitus desarrollada por Pierre Bourdieu.

- CE3. Los trabajos son recensiones, editoriales o erratas.

\subsection{Ajuste y creación de base de datos bibliográfica}

Una vez depurada la producción científica se trabajó con 104 documentos (ver Apendice). Se elaboró una base de datos en el software Excel con los siguientes campos: 1) autor, 2) título del trabajo, 3) año, 4) datos de la fuente (nombre de la revista/libro, volumen, año, número de artículo, páginas, DOI, resumen, palabras clave, referencias, editorial), 4) país, 5), idioma, 6) tipo de documento, y 7) tipo de acceso. Finalmente, se asignó a cada documento un identificador secuencial.

\subsection{Análisis de la información}

Finalmente se realizó el análisis de la información considerando dos etapas, En la primera se cuantificó la producción científica utilizando como criterios la cantidad de trabajos por año, el idioma, país, tipo de publicación, tipo de acceso, e impacto por número de citas. En la segunda, se llevó a cabo una lectura a profundidad de los resúmenes y en algunos casos de los documentos completos para identificar las líneas de investigación desde las que se ha abordado el estudio del tema, para lograrlo, se importó la base de datos diseñada en Excel al software VOSViewer, que es una herramienta que ha sido ampliamente utilizada para realizar análisis bibliométricos (Bornmann et al., 2016; Moreno, 2019; Peirats et al., 2019; Rodríguez et al., 2019). Lo anterior permitió extraer los resúmenes de los trabajos seleccionados, y con ellos crear mapas semánticos que permitieran más fácilmente delimitar las tendencias investigativas. 


\section{Resultados}

\subsection{Evolución de la cantidad de trabajos científicos publicados entre 2009 y 2019}

La evolución de la publicación de los trabajos científicos fue en aumento hasta el 2015, año en el que se publicaron 17 documentos (16.35\%), misma cantidad que en 2017, a partir de 2018 existe un decaimiento en la producción. En la Figura 1 se observa la tendencia diacrónica en Scopus y WOS. Es necesario mencionar que la investigación no consideró las publicaciones del 2020 debido a que al terminar la redacción de este documento aún no concluía el año, por lo que se puede inferir que existen documentos que abordan el mismo tema.

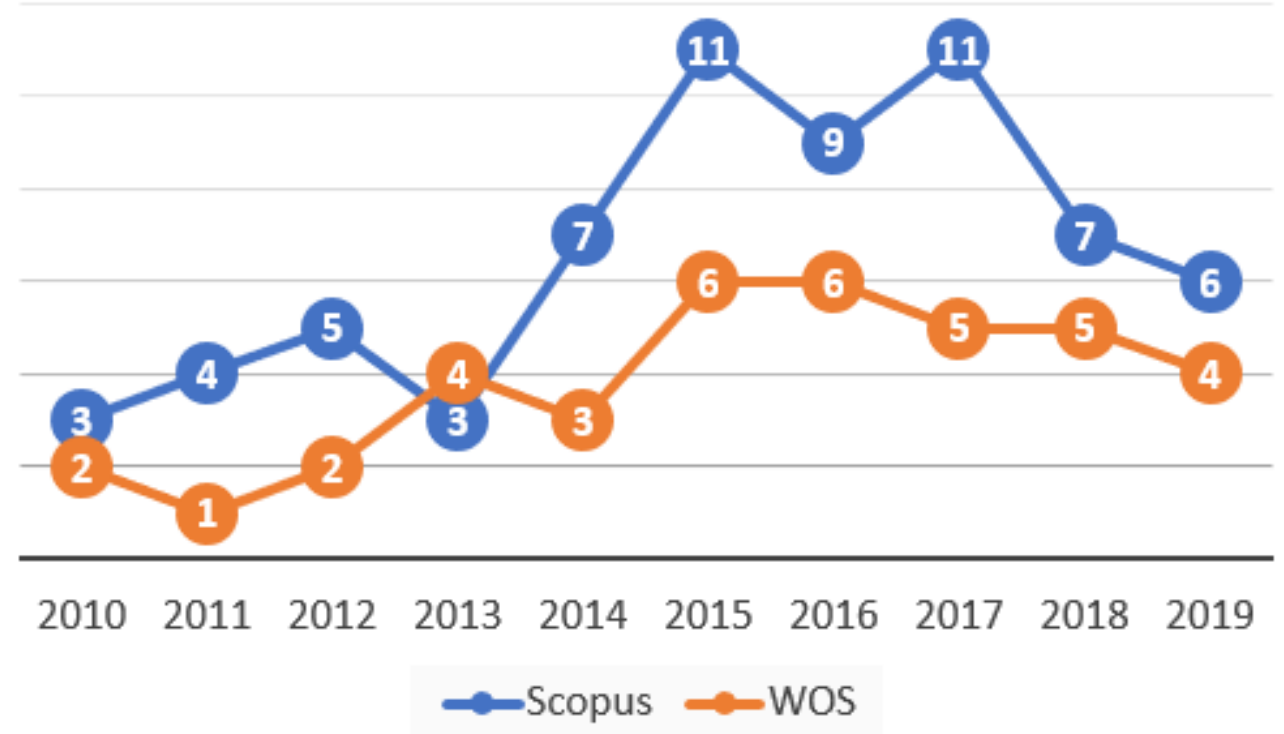

Figura 1. Tendencia de producción científica en WOS y Scopus sobre campo, habitus y capital.

\subsection{Producción científica por idioma y país}

En la Figura 2 puede observarse la distribución geográfica de la producción científica sobre campo, capital y habitus, esta proviene en gran parte de Reino Unido con 32 publicaciones (30.77\%), seguido por Australia con $11(10.58 \%)$ y Estados Unidos con $10(9.62 \%)$, lo que representa que estos países han producido un poco más de la mitad (50.97\%) de la producción mundial respecto al tema. El inglés es el lenguaje más utilizado con un predominio de $97(93.7 \%)$ publicaciones realizadas en este idioma, seguidas muy de lejos por el portugués con $4(3.85 \%)$. Existe un documento en croata, ruso y español respectivamente, la Tabla 3 especifica el idioma de cada documento revisado. 
35

30

25

20

15

10

5

0

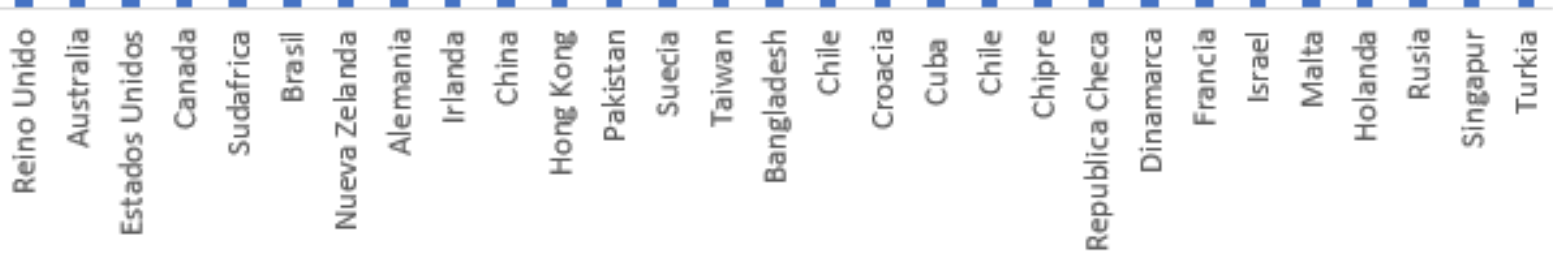

Figura 2. Producción científica por país.

Tabla 3

Documentos por idioma

\begin{tabular}{|l|l|l|l|}
\hline $\begin{array}{l}\text { Idioma } \\
\text { Ingles }\end{array}$ & Total & Porcentaje & Publicaciones \\
& 97 & $93.7 \%$ & $1,2,3,4,5,6,7,8,9,10,12,13,14,15$, \\
& & $16,17,18,19,20,21,22,23,24,25,26$, \\
& & $27,28,29,30.31,32,33,34,35,36,37$, \\
& & $38,39,40.41,42,43,44,45,46,47,48$, \\
& & $49,50,51,52,53,54,55,56,57,58,59$, \\
& & $60,61,62,63,64,65,66,67,68,69,70$, \\
& & $71,72,73,74,75,76,77,78,79,80,81$, \\
Portugués & & $82,83,84,85,86,87,88,89,90,91,92$, \\
Croata & & $93,96,98,101,102,103$. \\
Ruso & 4 & $9.85 \%$ & $94,95,97,100$ \\
Español & 1 & $0.96 \%$ & 99 \\
\hline & 1 & $0.96 \%$ & 104 \\
\hline
\end{tabular}




\subsection{Tipos de producto y accesibilidad para los lectores}

La producción científica puede categorizarse como artículos, libros, capítulos de libro, revisiones y documentos de conferencias, en la Tabla 4 puede observarse la distribución, destacando que los artículos en revistas tienen una mayor difusión con 90 publicaciones $(86.54 \%)$.

\section{Tabla 4}

Documentos por tipo de publicación

\begin{tabular}{l|l|l|l|}
\hline Idioma & Total & Porcentaje & Publicaciones \\
Artículos & 90 & $86.54 \%$ & $1,2,3,4,5,6,7,8,10,12,11,13,14$, \\
& & & $15,16,17,18,19,20.21,22,23,24$, \\
& & $25,26,27,28,29,31,32,33,35,37$, \\
& & $39,40.41,42,43,44,46,47,48,49$, \\
& & $50,51,52,53,54,55,57,59,61,62$, \\
& & & $63,64,65,66,67,68,69,71,73,74$, \\
Capítulos de libro & & & $75,76,77,78,79,80,81,82,83,84$, \\
Libros & & & $85,86,87,88,90,91,92,93,94,95$, \\
Revisiones & 5 & $3.81 \%$ & $96,97,98,99,102,103,104$. \\
Documentos de conferencia & 4 & $2.85 \%$ & $30,36,45,72,89$ \\
& 3 & $1.92 \%$ & $9,34,38,58$, \\
& & & $60,100,101$ \\
\end{tabular}

Por otra parte, el balance en cuanto al tipo de acceso favorece a las publicaciones de tipo cerrado o de pago con $92(88.46 \%)$, mientras que aquellas en formato abierto son $12(11.54 \%)$. En la Tabla 5 se categorizan los trabajos de acuerdo con las restricciones para su consulta a texto completo.

\section{Tabla 5}

Documentos por tipo de acceso

\begin{tabular}{|l|l|l|l|}
\hline Tipo de acceso & Total & Porcentaje & Publicaciones \\
Cerrado & 92 & $88.46 \%$ & $1, \ldots, 92$ \\
Abierto & 12 & $11.54 \%$ & $93, \ldots, 104$ \\
Total & 104 & $100 \%$ & \\
\hline
\end{tabular}

Se identificaron las revistas de acceso abierto y cerrado con más publicaciones. En acceso cerrado la revista British Journal Of Sociology of Education tiene el mayor número de documentos con 4, mientras que en acceso abierto Educacao e pesquisa tiene 2. Como se puede observar en la Tabla 6, de estas revistas, 4 se encuentran clasificadas en el primer cuartil de Scientific Journal Rankings, una en el segundo cuartil, y 5 en el tercer cuartil, el índice $\mathrm{H}$ menor es de 7 y el mayor de 64.

\section{Tabla 6}

Clasificación de revistas por SJR e índice $\mathrm{H}$.

\begin{tabular}{|c|c|c|c|c|c|c|c|}
\hline \multicolumn{4}{|l|}{ Acceso abierto } & \multicolumn{4}{|l|}{ Acceso cerrado } \\
\hline Revista & Núm. & SJR & $\mathrm{H}$ & Revista & Núm. & SJR & $\mathrm{H}$ \\
\hline Educacao e pesquisa & 2 & Q3 & 13 & British Journal Of Sociology Of Education & & Q1 & 64 \\
\hline Cogent education & 1 & Q3 & 7 & $\begin{array}{l}\text { Higher Educacion Research And } \\
\text { Development }\end{array}$ & 3 & Q1 & 51 \\
\hline Cogent Social Sciences & 1 & Q3 & 9 & Critical Studies in Education & 3 & Q1 & 27 \\
\hline Egitim Ve Bilim & 1 & Q3 & 16 & Sociological Research Online & 2 & Q2 & 41 \\
\hline $\begin{array}{l}\text { Policy Futures In } \\
\text { Education }\end{array}$ & 1 & Q3 & 12 & Sport Education And Society & 2 & Q1 & 55 \\
\hline
\end{tabular}




\subsection{Trabajos de mayor impacto internacional}

De acuerdo con la información obtenida de las bases de datos de SCOPUS y WOS, el mayor número de citas para un trabajo en acceso abierto es de 19 (trabajo 96), mientras que en acceso cerrado es de 81 (trabajo 48). Se puede observar que $25(24.04 \%)$ de los documentos no han sido citados por otros científicos, mientras que $12(11.54 \%)$ solo ha sido citado una vez. La Tabla 7 muestra la relación de las publicaciones y su número de citas.

\section{Tabla 7}

Impacto por número de citas por cada publicación

\begin{tabular}{|l|l|l|}
\hline $\begin{array}{l}\text { Total de } \\
\text { citas }\end{array}$ & Número de publicación & Frecuencia \\
81 & 48 & 1 \\
70 & 6 & 1 \\
57 & 79 & 1 \\
51 & 35 & 1 \\
39 & 81 & 1 \\
27 & 62 & 1 \\
26 & 37,90 & 2 \\
23 & 59 & 1 \\
22 & 96 & 1 \\
20 & 60,88 & 2 \\
19 & $13,16,22,84$, & 4 \\
18 & 5 & 1 \\
17 & 57 & 1 \\
16 & 15 & 1 \\
15 & 27,75 & 2 \\
14 & 4,8 & 2 \\
13 & 52,63 & 2 \\
12 & 66 & 1 \\
11 & 42,67 & 2 \\
10 & 83 & 1 \\
9 & $14,18,28,91$ & 4 \\
8 & $2,17,53,94$ & 4 \\
7 & $10,47,51,61$ & 4 \\
6 & $26,54,71$ & 3 \\
\hline & $39,43,76$ & 3 \\
4 & $3,12,, 19,64,73,95$ & 6 \\
3 & $9,30,31,33,40,55,58,68,82,86,98$, & 11 \\
2 & $23,25,85,102$, & 4 \\
1 & $11,20,21,29,32,38,41,50,78,80,97,101$ & 12 \\
0 & $1,7,24,34,36,44,45,46,49,56,65,69,70,72,74,75,77,87,89,92,93,99,100$, \\
& $103,104$. & 25 \\
\hline
\end{tabular}

En cuanto al impacto de citas por país, Reino Unido encabeza la lista con 204 citas seguido por Australia que contabiliza 108, Canadá 92, Estados Unidos 82, Sudáfrica 56 y Nueva Zelanda con 42. 29 países aportan en conjunto 226 citas. En la Figura 3 puede observarse la distribución por país, así como la colaboración entre las publicaciones. 


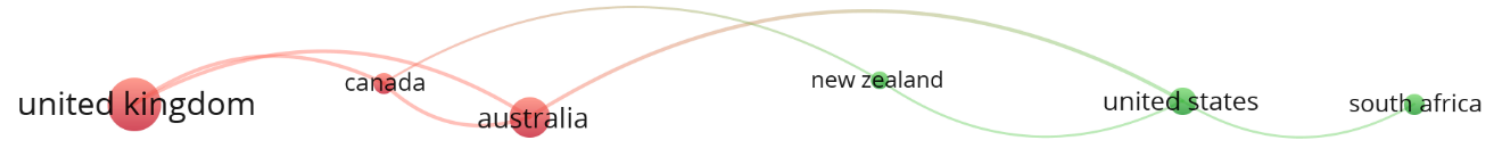

\& Vosviewer

Figura 3. Colaboración científica por país.

\subsection{Líneas de investigación sobre campo, capital y habitus}

El análisis de las líneas de investigación se llevó a cabo en dos etapas, primero se examinaron las palabras clave de los 104 artículos seleccionados, con esa información se pudo inferir cuáles son las tendencias actuales en el estudio del tema. Entre las palabras clave con más coocurrencias (número de veces que se repite un término) se encuentran habitus (25), higher education (19), capital (13), cultural capital (12) y field (12). En la Figura 4 se muestra gráficamente la relación de ocurrencias de oalabras clave, mientras que en la Tabla 8 se muestra la relación completa y la fuerza de enlace total de cada una de ellas.

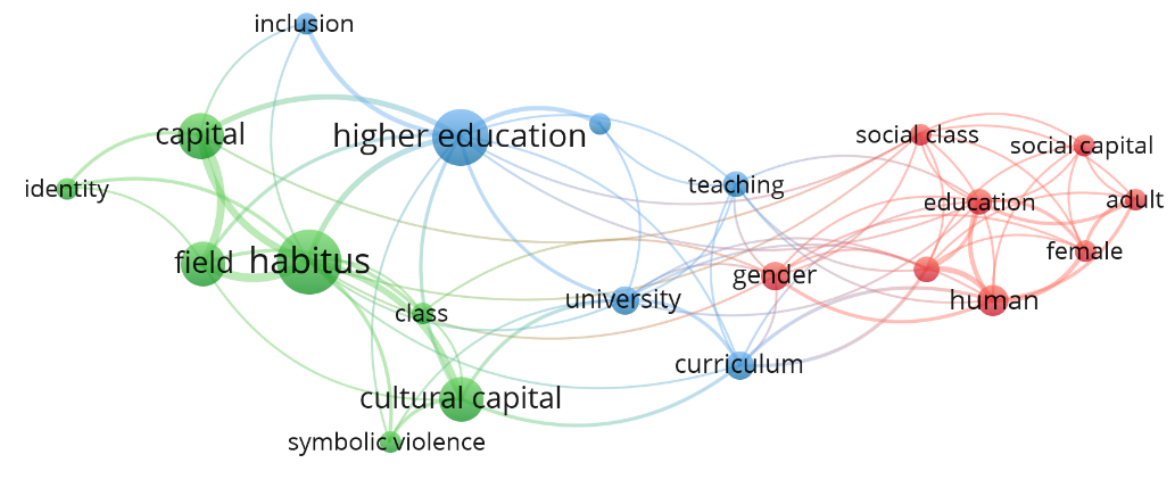

\section{VOSviewer}

Figura 4. Análisis de las palabras clave.

Al revisar los resúmenes de los documentos y en algunos casos de interés, el documento completo, se observó que existen dos arquetipos de líneas de investigación: la primera, en consolidación, en la que se integran 95 $(91.35 \%)$ documentos que representan las tendencias investigativas más trabajadas sobre el campo, el capital y el habitus, y la segunda, una línea emergente que agrupa 9 documentos $(8.65 \%)$ que aborda los conceptos mencionados desde enfoques del uso de la tecnología y metaanálisis conceptuales. Algunos trabajos sitúan su objeto de estudio en el campo de la música $(9,61)$ y los deportes $(4,28.35,66)$. En la Tabla 9 se especifican el conjunto de líneas de investigación identificadas. 
Tabla 8

Ocurrencias de palabras clave y fuerza de enlace total

\begin{tabular}{|l|l|l|}
\hline Palabra clave & Ocurrencias & Fuerza de enlace total \\
habitus & 25 & 33 \\
higher education & 19 & 25 \\
capital & 13 & 21 \\
cultural capital & 12 & 15 \\
field & 12 & 21 \\
human & 6 & 24 \\
curriculum & 5 & 15 \\
gender & 5 & 12 \\
university & 5 & 15 \\
education & 4 & 18 \\
humans & 4 & 18 \\
interview & 4 & 16 \\
teaching & 4 & 9 \\
adult & 3 & 10 \\
class & 3 & 5 \\
female & 3 & 12 \\
identity & 3 & 5 \\
inclusion & 3 & 5 \\
social capital & 3 & 6 \\
social class & 3 & 10 \\
students & 3 & 5 \\
symbolic violence & 3 & 6 \\
\hline
\end{tabular}

Tabla 9

Líneas de investigación en consolidación y emergentes

\begin{tabular}{|c|c|c|c|}
\hline \multirow{5}{*}{$\begin{array}{l}\text { Tipo } \\
\text { En consolidación }\end{array}$} & Línea & Documentos & Tota \\
\hline & $\begin{array}{llr}\begin{array}{l}\text { Elecciones } \\
\text { trayectorias }\end{array} \text { de } & \text { los } \\
\text { estudiantes } & & \end{array}$ & $\begin{array}{l}1,2,3,6,10,17,18,24,27,28,29 \\
30,31,32,33,40,41,44,46,47,50 \\
52,53,60,61,62,64,66,69,72,75 \\
77,78,86,91,92,95,98,99,101 .\end{array}$ & 40 \\
\hline & $\begin{array}{l}\text { Docentes y prácticas } \\
\text { de enseñanza }\end{array}$ & $\begin{array}{l}4,7,9,16,25,26,35,37,38,39,45, \\
54,63,68,74,76,80,84,85,87,89 \\
93,102,103 .\end{array}$ & 24 \\
\hline & $\begin{array}{l}\text { Empleo y capitales } \\
\text { para posicionarse en } \\
\text { el campo profesional. }\end{array}$ & $\begin{array}{l}5,8,13,14,15,20,21,34,42,43,48, \\
49,51,59,63,71,88,90 .\end{array}$ & 17 \\
\hline & $\begin{array}{l}\text { Género y clases } \\
\text { sociales. }\end{array}$ & $\begin{array}{l}12,19,55,57,65,67,73,79,81,82 \\
83,97,100,101\end{array}$ & 14 \\
\hline \multirow[t]{2}{*}{ Emergente } & $\begin{array}{l}\text { Campo del uso de } \\
\text { la tecnología en la } \\
\text { educación superior. }\end{array}$ & $11,22,23,56,70,96$ & 6 \\
\hline & $\begin{array}{l}\text { Metaanálisis de la } \\
\text { obra de Bourdieu }\end{array}$ & $36,58,94$ & 3 \\
\hline
\end{tabular}




\subsection{Líneas en consolidación}

En cuanto a las líneas en consolidación destacan los estudios acerca de la elección de los estudiantes para acceder o permanecer en la universidad (38.46\%), en los que se manifiesta que los estudiantes experimentan y conceptualizan posiciones para participar en el aprendizaje formal $(17,18,24,27,29,32,33,41,47,64,75)$, y con ello crean y transforman sus identidades aprendiendo a convertirse en alumnos $(2,69)$. Estas identidades invariablemente están ligadas a sus experiencias previas, antecedentes sociales y culturales, así como sus contextos formativos preuniversitarios $(40,66,72)$.

La segunda línea aborda la relevancia de las prácticas de enseñanza en diversos contextos universitarios $(23.08 \%)$, los estudios aquí agrupados, en general argumentan que las prácticas educativas están determinadas por la posición de los docentes en el campo de la educación superior $(45,63$, 98), y que la enseñanza se encuentra significativamente influenciada por las políticas implementadas desde la institución escolar (25) que incluso determinan los criterios de contratación, de evaluación, la garantía de calidad y los recursos de apoyo al aprendizaje $(101,102)$

Los estudios acerca de las aproximaciones para comprender la transición de la universidad al campo laboral se agrupan en una tercera línea de investigación (16.35\%), que contribuye en comprender de una forma más amplia las percepciones de los graduados universitarios respecto a los recursos que deben de desplegar (capital) para lograr incorporarse a las nuevas industrias nacidas en el auge de la sociedad del conocimiento $(5,8,13,20)$. Otros estudios examinan la manifestación del gerencialismo en la Educación Superior, que obliga a las instituciones educativa a evaluar exhaustivamente a los estudiantes mediante indicadores de desempeño para producir graduados empleables (42)

Finalmente, las investigaciones relacionadas con los estudios de genero y la categorización de las clases sociales conforman la última línea de investigación consolidada (13.46\%), los estudios revisados exploran las experiencias de las mujeres para acceder a la educación superior $(12,73,81)$, así como el aumento de la matriculación femenina en las universidades (97). Algunos autores concluyen que las madres de clase media procuran equilibrar su capital social, cultural y económico para mejorar la educación de sus hijas y facilitar su acceso a la educación superior (19).

\subsection{Líneas emergentes}

Entre las líneas emergentes, destacan el análisis del uso de las tecnologías en los escenarios universitarios, así como los habitus de los estudiantes universitarios en programas educativos mediados por herramientas digitales, o bien en modelos educativos duales (5.78\%). Estos estudios exploran el aprendizaje mediado digitalmente (23) y las prácticas sociales de los estudiantes, haciendo énfasis en la experiencia, brechas y oportunidades para acceder a dispositivos tecnológicos fuera de la institución escolar (56, 70). Uno de estos estudios utiliza el concepto habitus tecnológico (22), que en conjunto con el capital cultural incorporado (disposición y valores), el capital cultural objetivado (computadoras y teléfonos móviles), y el capital social (conexiones personales), condicionan las prácticas relacionadas con el acceso y valoración del uso de dispositivos digitales. La última línea la conforman metaanálisis conceptuales de la obra de Pierre Bourdieu (2.88\%). Al analizar el conjunto de documentos recuperados con el software VOSViewer, la Figura 5 confirma que las principales líneas de investigación se desarrollan en torno al estudiante y sus trayectorias, la escuela y las prácticas de enseñanza, y la transición entre la universidad y el campo laboral.

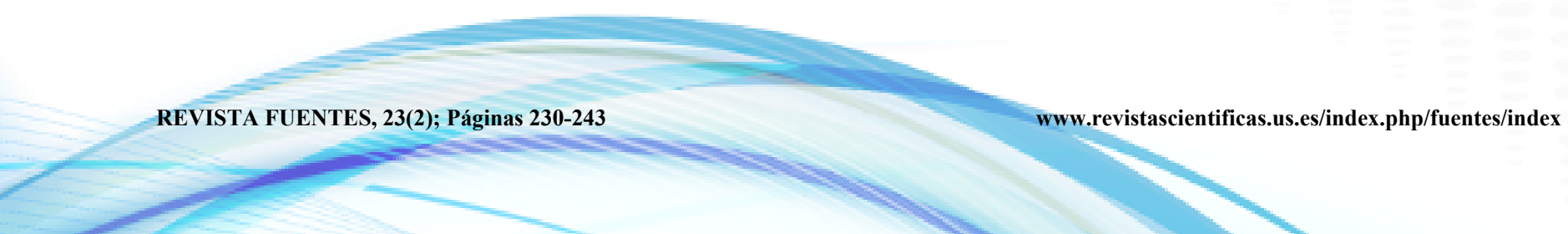




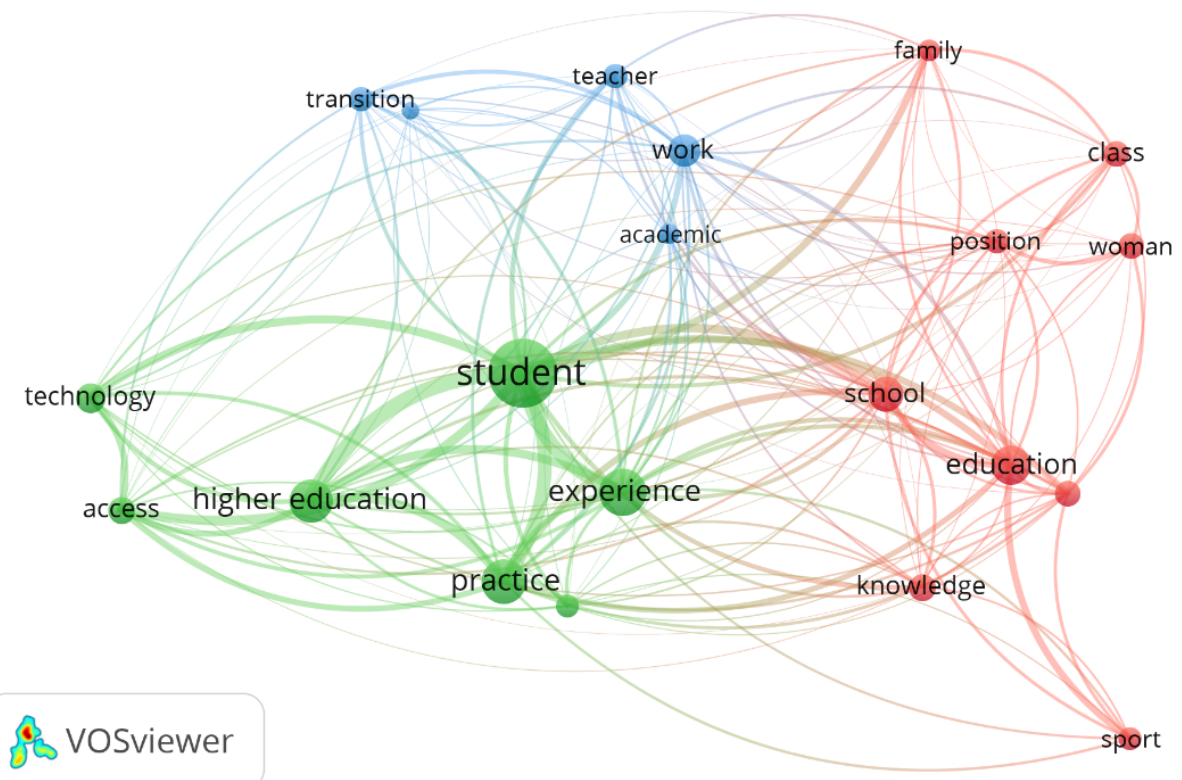

Figura 5. Líneas de investigación sobre campo, habitus y capital.

\section{Discusión y conclusiones}

El mapeo sistematico de la literatura realizado en este artículo tuvo como propósito brindar a los estudiantes e investigadores en el tema una ruta de exploración que les permita tomar decisiones investigativas sobre la base de las tendencias en la producción científica en el tema de las prácticas sociales, en especial en aquellas fundamentadas en la estructura conceptual de campo, capital y habitus. El estudio representa una orientación general acerca de la construcción de un proceso de selección de literatura de alto impacto internacional, que sin embargo requiere de una revisión más profunda en la que se elijan nuevos criterios de inclusión y exclusión de materiales bibliográficos, así como de orientaciones contextuales que incluyan la exploración de otros niveles educativos.

La fortaleza de la investigación radica en la selección de una metodología de mapeo sistemático ampliamente utilizada en el campo de las ciencias sociales, que ofrece la posibilidad de tener una visualización general sobre una vasta producción académica de forma ordenada, ya que fue acotada al periodo 2009-2019, además incluyó publicaciones en revistas científicas, libros y actas de congresos con reconocimiento de calidad internacional. Se encontraron diferencias significativas en el idioma de publicación, ya que el inglés dominó de sobremanera sobre otras lenguas, de igual forma los países con mayor producción fueron el Reino Unido y Australia, seguidos por países como Estados Unidos, Canadá y Sudáfrica. Lo anterior puede indicar que la apropiación de la teoría social de Bourdieu ha tenido una mayor acogida en países angloparlantes.

Se identificaron al menos 5 líneas de investigación que asocian los conceptos teórico-metodológicos de Bourdieu con la Educación Superior: 1) las elecciones de los estudiantes para elegir una carrera profesional y su trayectoria después de haber ingresado a la universidad, 2) los docentes y sus prácticas de enseñanza dominadas por los preceptos de la institución escolar, 3) la distribución desigual de los capitales para posicionarse en el empleo profesional, 4) las diferencias de género y la estratificación de clases sociales en la universidad, y 5) los capitales para hacer uso eficiente de la tecnología en la Educación Superior.

Este documento permite al lector establecer retos de exploración investigativa, sin embargo se debe hacer notar que tiene al menos dos limitantes, la primera está relacionada con las bases de datos que se eligieron para buscar la información, si bien Scopus y WOS se han consolidado como herramientas para localizar producción científica de alta calidad en el mundo, no incluyen al cúmulo de revistas certificadas y validadas por su impacto internacional por organismos nacionales, por ejemplo el Consejo Nacional de Ciencia y Tecnologia (CONACYT, México), la Fundación Española para la Ciencia y Tecnología (FECYT, España) y el Consejo Nacional de Investigaciones Científicas y Técnicas (CONICET, Argentina), estos organismos tienen sistemas de clasificación de producción investigativa de alta calidad. 
Por otra parte, la segunda limitante se encuentra en el hecho de que para dar respuesta a las preguntas de investigación planteadas en el artículo se trabajó con los metadatos de la literatura, así como con las palabras clave, con los resúmenes de los documentos, y en algunos casos con la lectura completa de los artículos de mayor interés para este autor. De esta forma, queda como una tarea pendiente ofrecer detalles de cada uno de los hallazgos bibliográficos encontrados.

Finalmente, en este articulo se deja una invitación para que aquellos lectores interesados en el tema de las prácticas sociales desde el enfoque del campo, capital y habitus amplíen las preguntas de investigación y con ellas realicen nuevas revisiones sistemáticas de la literatura, o bien efectúen adecuaciones al método aquí presentado con el fin de provocar hallazgos que verifiquen o refuten los resultados encontrados en este documento.

\section{A. Apéndice}

La bibliografía mapeada puede revisarse en:

https://drive.google.com/file/d/1hw_sB9iT4jD7SofBFtbjNy3aN78FWTu2/view?usp=sharing

\section{Apoyos}

Consejo Nacional de Ciencia y Tecnologia (CONACYT). México.

\section{Referencias}

Alonso, L. (1930). Entre la bourdemanía y la reconstrucción de la sociología europea. Revista Española de Investigaciones Sociológicas, 97, 9-28.

Bonello, M., Wright, J., Morris, J., y Sadlo, G. (2018). Bourdieu and interprofessional education: what's the relevance? Studies in Continuing Education, 40(1), 1-16. Retrieved from https://dx.doi.org/10.1080/0158037x $.2017 .136026910 .1080 / 0158037 \times .2017 .1360269$

Bornmann, L., Thor, A., Marx, W., y Schier, H. (2016). The application of bibliometrics to research evaluation in the humanities and social sciences: An exploratory study using normalized Google Scholar data for the publications of a research institute. Journal of the Association for Information Science and Technology, 67(11), 2778-2789. Retrieved from https://dx.doi.org/10.1002/asi.23627 10.1002/asi.23627

Bourdieu, P. (1998). La distinción. Criterio y bases sociales del gusto. Taurus.

Bourdieu, P. (2001). Poder, derecho y clases sociales. Desclée de Brower.

Bourdieu, P. (2012). Bosquejo de una teoría de la práctica. Prometeo Libros.

Bourdieu, P., y Passeron, J. (1996). La reproducción. Elementos para una teoría de la enseñanza. Fontamara.

Bourdieu, P., y Wacquant, L. (2005). Una invitación a la sociología reflexiva. Siglo XXI Editores.

Bustamante, G. (2016). Sobre el concepto de campo en Bourdieu. Magis, 9(18), 49-66.

Casillas, M., Ramírez, A., y Ortiz, V. (2014). El capital tecnológico: una nueva especie de capital cultura.

Háblame de TIC: Tecnología digital en la Educación Superior, 23-38.

Cerón, A. (2012). Habitus y capitales: ¿Disposiciones o dispositivos sociales? Notas teórico-metodológicas para la investigación social. Revista Latinoamericana de Metodología de la Investigación Social, 4, 68-82.

Cerón-Martínez, A. U. (2019). Habitus, campo y capital. Lecciones teóricas y metodológicas de un sociólogo bearnés. Cinta de moebio, 66(66), 310-320. Retrieved from https://dx.doi.org/10.4067/s0717 $-554 \times 201900030031010.4067 /$ s0717-554×2019000300310

De-Las-Heras, C., Martel, T., y Jambrino, C. (2018). Análisis de las redes académicas y tendencias científicas de la comunicación en las universidades españolas. Revista Prisma Social, 22, 229-246.

Delgado, E., y Repiso, R. (2013). El impacto de las revistas de comunicación: comparando Google Scholar Metrics, Web of Science y Scopus. Comunicar, 41, 45-52.

Giménez, G. (1999). La sociología de Pierre Bourdieu en Perspectivas teóricas contemporáneas de las ciencias sociales, Proyecto Antología de teoría sociológica contemporánea. UNAM/FCPyS.

González, V. C., Morales, L. D. G., y Montoya, M. S. R. (2019). Comportamiento métrico sobre evaluación de la educación en emprendimiento. Investigación Bibliotecológica: archivonomía, bibliotecología e información, 33(79), 99-99. Retrieved from https://dx.doi.org/10.22201/iibi.24488321xe.2019.79.57902 10 .22201 iibi.24488321xe.2019.79.57902

Grenfell, M. (2012). Pierre Bourdieu Key Concepts. Routledge.

Hart, C. (2019). Education, inequality and social justice: A critical analysis applying the Sen-Bourdieu Analytical Framework. Policy Futures in Education, 17(5), 582-598.

Ignatow, G., y Robinson, L. (2017). Pierre Bourdieu: theorizing the digital. Information, Communication \& 
Society, 20(7), 950-966. Retrieved from https://dx.doi.org/10.1080/1369118x.2017.1301519 10.1080/1369118x .2017.1301519

Lee, K., y Chen, W. (2017). A long shadow: Cultural capital, techno-capital and networking skills of college students. Computers in human behavior, 70, 67-73.

López-Meneses, E., Vázquez-Cano, E., y Román-Graván, P. (2015). Analysis and Implications of the Impact of MOOC Movement in the Scientific Community: JCR and Scopus (2010-13). Comunicar, 22, 73-80. Retrieved from https://dx.doi.org/10.3916/c44-2015-08 10.3916/c44-2015-08

M, C. (2018). Comprender a Bourdieu: las estrategias sociales de capitalización. Revista Colombiana de Sociología, 41(2), 219-237.

Moreno, A. (2019). Estudio bibliométrico de la producción científica en Web of Science: Formación Profesional y blended learning. Píxel-Bit. Revista de Medios y Educación, 56, 149-168. https://doi.org/10.12795/pixelbit .2019.i56.08

Peirats, J., Marín, D., y Vidal, M. (2019). Bibliometría aplicada a la gamificación como estrategia digital de aprendizaje. RED. Revista de Educación a Distancia, 60. http://dx.doi.org/10.6018/red/60/05

Pérez, M., y Vladimirovna, N. (2017). La producción científica sobre la innovación social para el desarrollo local: una revisión bibliométrica la estructura y la evolución del campo de dominio científico. Prisma Social. Revista de Ciencias Sociales, 19, 146-182. Retrieved from https://revistaprismasocial.es/article/view/1750

Petersen, K., Robert, F., Shahid, M., y Mattsson, M. (2008). Systematic Mapping Studies in Software Engineering. EASE, 8, 68-77.

Ramírez, M., y García, F. (2018). Co-creación e innovación abierta: Revisión sistemática de literatura. Comunicar, 26(54), 9-18.

Rodríguez-García, A.-M., Raso-Sánchez, F., y Ruiz-Palmero, J. (2019). Competencia digital, educación superior y formación del profesorado: un estudio de meta-análisis en la web of science. Pixel-Bit, Revista de Medios y Educación, 65-82. https://dx.doi.org/10.12795/pixelbit.2019.i54.04

Salado, L., Velásquez, M., y Ochoa, R. (2016). La apropiación de las TIC en los estudiantes universitarios: Una aproximación desde sus habitus y representaciones sociales. Estudios LAMBDA. Teoría y práctica de la didáctica de la lengua y literatura, 1(1), 215-234. https://doi.org/10.36799/el.v1i1.32

Singh, H., Andrade, A. D., y Techatassanasoontorn, A. A. (2018). The practice of ICT-enabled development. Information Technology for Development, 24(1), 37-62. Retrieved from https://dx.doi.org/10.1080/02681102 $.2017 .128328410 .1080 / 02681102.2017 .1283284$

Wacquant, L. (2018). Bourdieu Comes to Town: Pertinence, Principles. Applications. International Journal of Urban and Regional Research, 42(1), 90-105. 